\title{
Mathematical Model and Optimal Control of New-Castle Disease (ND)
}

\author{
Uwakwe Joy Ijeoma ${ }^{1}$, Inyama Simeon Chioma ${ }^{2,}$, , Omame Andrew ${ }^{2}$ \\ ${ }^{1}$ Department of Mathematics, Alvan Ikoku Federal College of Education, Owerri, Nigeria \\ ${ }^{2}$ Department of Mathematics, Federal University of Technology, Owerri, Nigeria
}

Email address:

Joychi13@yahoo.com (U. J. Ijeoma), scinyama2011@yahoo.com (I. S. Chioma), omame2020@gmail.com (O. Andrew)

${ }^{*}$ Corresponding author

To cite this article:

Uwakwe Joy Ijeoma, Inyama Simeon Chioma, Omame Andrew. Mathematical Model and Optimal Control of New-Castle Disease (ND). Applied and Computational Mathematics. Vol. 9, No. 3, 2020, pp. 70-84. doi: 10.11648/j.acm.20200903.14

Received: December 9, 2019; Accepted: January 10, 2020; Published: June 4, 2020

\begin{abstract}
We formulated a five compartmental model of ND for both the ordinary and control models. We first determined the basic Reproduction number and the existence of Steady (Equilibrium) states (disease-free and endemic). Conditions for the local stability of the disease-free and endemic steady states were determined. Further, the Global stability of the disease-free equilibrium (DFE) and endemic equilibrium were proved using Lyponav method. We went further to carry out the sensitivity analysis or parametric dependence on $\mathrm{R}_{0}$ and later formulated the optimal control problem. We finally looked at numerical Results on poultry productivity in the presence of Infectious Newcastle Disease (ND) and we drew six graphs to demonstrate this. We observe that in absence of any control measure, the number of latently infected birds will increase rapidly from the initial population size of 80 to 160 birds within 1-3 days, whereas in the presence of control measures the population size will reduces to about 30 birds and goes to a stable state. This shows that the control measures are effective. The effect of the three control measures on the infectious classes can be seen. The number of non-productive infectious birds reduces to zero with control whereas the number of infectious productive reduces to about 8 birds and goes to its stable state when control is applied. This shows that the application of all three control measures tends to be more effective in the non- productive infectious bird population. It was also establish that the combination of efficient vaccination therapy and optimal efficacy of the vaccines are significantly more effective in the infectious productive birds' population, since the combination reduces the population size of the birds to zero with 9-10 days. From the simulation also we see that optimal efficacy of the vaccine and effort to increase the number of recovered birds increases the number of latently infected birds population to about 129 at the early days of the infection whereas from another graph, the infectious productive birds reduces to 15 while the non -productive birds reduces to zero. The results from the simulation also show clearly, the effect of vaccination therapy on the latently infected birds. We observe that this programme will reduce the number of latently infected birds even if it not done more often. From the simulation, we further observe that this programme has effect on the infectious classes especially the non-productive infectious bird population, which reduces to zero after about 4 days.
\end{abstract}

Keywords: Newcastle Disease (ND), Disease-free Equilibrium (DFE), Global Stability, Efficient Vaccination, Lyponav Method

\section{Introduction}

Newcastle disease (ND) is a contagious viral infection from a member of the family Paramyxoviridae in the genus Avulavirus which causes respiratory nervous disorder in several species of birds including chickens and turkeys. There are ten serotypes of avian paramyxo viruses designated
APMV-I to APMV-10 and ND virus (NDV) has been designated APMV-1. NDV has also been categorized into five pathotypes based on clinical signs in infected chickens, designated: a) viscerotropic velogenic, b) neurotropic velogenic, c) mesogenic, d) lentogenic or respiratory and e) asymptomatic.

ND has a proven ability to spread internationally and cause major outbreaks. It undoubtedly is a threat to the Pacific region 
that quarantine authorities should be aware of. Potential methods of introducing the disease include infected day old chicks, frozen carcasses and contaminated feed or equipment. Wild and caged birds have played a major role in international spread on ND; [7]. Wild birds constitute a natural reservoir of low-virulence viruses, while poultry are the main reservoir of virulent strains. The most virulent form of ND virus causes up to 100 percent mortality in affected flocks, [8].

The most severe strain is the viscerotropic velogenic Newcastle disease. It is often called Exotic Newcastle Disease' and infection of susceptible bird with this form usually causes high mortality. The milder form of the disease is the 'mesogenic ND'. Humans may become infected; manifested by unilateral or bilateral reddening, excessive lachrymation, oedema of the eyelids, conjunctivitis and subconjunctival haemorrhage. Velogenic NDV is endemic in areas of Mexico, Central and South America, widely spread in Asia, the Middle East and Africa, and in double-crested wild cormorants in the US and Canada. Lentogenic strains of NDV are worldwide in their distribution while widespread mesogenic pathotypes with a special adaptation to pigeons (that is, pigeon paramyxovirus) do not appear to infect other poultry readily, [3]. Other papers consulted in this work are: $[1,2,4,5,6,9,10,11,12,13,14,15,16,17,18,19,20,21$, $22,23,24,25,26,27,28,29$ and 30].

\section{Method}

\subsection{Assumptions of the Model}

1. The productive birds becomes non-productive as a result of the disease.

2. Birds are recruited by immigration or birth.

3. Birds are recruited only to the non-productive susceptible class.

4. Infected birds can be treated.

5. Poultry birds that recover from one strain can become susceptible to anothstrain.

6 . Recovered birds move only to the non-productive susceptible class.

\subsection{Model Parameters for Newcastle Disease}

Table 1. Symbols and Parameter.

\begin{tabular}{ll}
\hline Symbols/Parameters & Interpretation \\
\hline $\mathrm{N}(\mathrm{t})$ & Total bird population at time $\mathrm{t}$ \\
$\mathrm{S}_{\mathrm{P}}$ & Susceptible productive birds at time t \\
$\mathrm{S}_{\mathrm{n}}$ & Susceptible non-productive birds at time \\
$\mathrm{I}_{\mathrm{p}}$ & Infective productive birds at time $\mathrm{t}$ \\
$\mathrm{I}_{\mathrm{n}}$ & Infective non-productive birds at time $\mathrm{t}$ \\
$\mathrm{R}$ & Recovered birds at time $\mathrm{t}$ \\
$\rho$ & Recruitment rate of birds \\
$\mathrm{a}$ & Progression rate from non-productive susceptible to productive susceptible \\
$\beta n$ & Contact rate for non-productive susceptible birds \\
$\beta_{p}$ & Contact rate for productive susceptible birds \\
$\delta$ & Recovery rate \\
$\mathrm{h}$ & Rate at which infective productive birds becomes non-productive \\
$\gamma$ & Loss of immunity rate \\
$\mathrm{d}$ & Natural death rate of birds \\
$\mu$ & Disease-induced death rate for infected birds \\
$\mathrm{u}(\mathrm{t})$ & Efficiency of vaccine therapy in preventing new infection \\
$\mathrm{v}(\mathrm{t})$ & Efficiency of drug therapy in inhibiting the virus strain \\
\hline
\end{tabular}

\subsection{Model Flow Diagram}

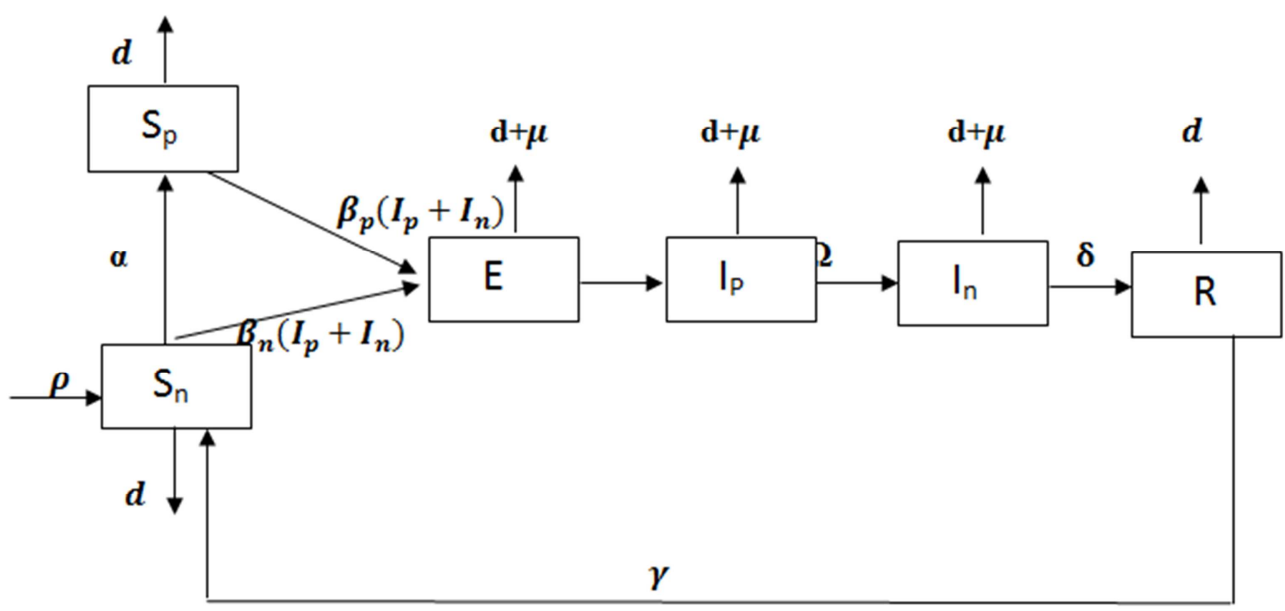

Figure 1. Flow diagram for Newcastle Disease. 


\subsection{Mathematical Model for Newcastle Disease}

Following the flow diagram we obtain the following system of ordinary differential equations.

$$
\left.\begin{array}{l}
\frac{d S_{n}}{d t}=\rho-\beta_{n}\left(I_{p}+I_{n}\right) S_{n}-(a+d) S_{n}+\gamma R \\
\frac{d S_{p}}{d t}=a S+\beta_{p}\left(I_{p}+I_{n}\right) S_{p}-d S_{p} \\
\frac{d I_{n}}{d t}=\beta_{n}\left(I_{p}+I_{n}\right) S_{n}+h I_{p}-(\mu+\delta+d) I_{n} \\
\frac{d I_{p}}{d t}=\beta_{p}\left(I_{p}+I_{n}\right) S_{p}-(h+\mu+d) I_{p} \\
\frac{d R}{d t}=(\mu+\delta) I_{n}-(\gamma+d) R
\end{array}\right\}
$$

\subsection{Control Measure}

Two control measures are employed which are; $u(t)$ the efficiency of the vaccine therapy in preventing the infection, considering the need for a revaccination program to maintain adequate protection from the disease $\mathrm{U}(\mathrm{t})$ and $\mathrm{V}(\mathrm{t})$ the efficiency of drug in inhibiting the virus strain. There are different strains of the Newcastle disease in birds; some are more severe than the other such as the viscerotropic velogenic strain, characterized by high mortality rate amongst birds.

The control model is given as:

$$
\left.\begin{array}{l}
\frac{d S_{n}}{d t}=\rho\left(1-U S_{n}\right)-\beta_{n}\left(I_{p}+I_{n}\right)(1-V) S_{n}-(a+d) S_{n}+\gamma R \\
\frac{d S_{p}}{d t}=a S_{n-\beta_{p}}\left(I_{p}+I_{n}\right)(1-V) S_{p}-d S_{p} \\
\frac{d I_{n}}{d t}=\beta_{n}\left(I_{p}+I_{n}\right)(1-V) S_{n}+h I_{p}-(\mu+\delta+d) I_{n} \\
\frac{d I_{p}}{d t}=\beta_{p}\left(I_{p}+I_{n}\right)(1-V) S_{p}-(h+\mu+d) I_{p} \\
\frac{d R}{d t}=\delta I_{n}-(\gamma+d) R
\end{array}\right\}
$$

\section{Results}

\subsection{Existence of Steady (Equilibrium) States}

The basic reproduction number $\mathrm{R}_{0}$ for the Newcastle disease model (1) is calculated using the Next Generation Operator method as described in (1).

Where

$$
F=\left(\begin{array}{ccc}
0 & \beta_{n} \frac{\rho}{\alpha+d}+\beta_{p} \frac{\alpha \rho}{(\alpha+d) \alpha} & \beta_{n} \frac{\rho}{\alpha+d}+\beta_{p} \frac{\alpha \rho}{(\alpha+d) \alpha} \\
0 & 0 & 0 \\
0 & 0 & 0
\end{array}\right)
$$

and

$$
V=\left(\begin{array}{ccc}
\omega+\mu+d & 0 & 0 \\
-\omega & \Omega+\mu+d & 0 \\
0 & \Omega & \mu+d+\delta
\end{array}\right)
$$

Thus,

$$
\begin{gathered}
F V^{-1}=\left(\begin{array}{c}
\frac{\left(\beta_{n} \rho+\beta_{p} \alpha \rho\right) \Omega \omega}{d(\alpha+d)(\omega+\mu+d)(\Omega+\mu+d)(\mu+d+\delta)}+\frac{\left(\beta_{n} \rho+\beta_{p} \alpha \rho\right)(\mu \omega+d \omega+\delta \omega)}{d(\alpha+d)} \\
0 \\
0
\end{array}\right) \\
F V^{-1}=\left(\begin{array}{ccc}
\frac{\left(\beta_{n} \rho+\beta_{p} \alpha \rho\right)}{d(\alpha+d)(\mu+d+\delta)} & \frac{\left(\beta_{n} \rho+\beta_{p} \alpha \rho\right)}{d(\alpha+d)(\Omega+\mu+d)}+\frac{\left(\beta_{n} \rho+\beta_{p} \alpha \rho\right)}{d(\alpha+d)(\Omega+\mu+d)(\mu+d+\delta)} \\
0 & 0
\end{array}\right)
\end{gathered}
$$


Therefore the basic reproduction, $\mathrm{R}_{0}$, which is the dominant eigenvalue, is given by,

$$
\mathrm{R}_{0}=\frac{\left(d \beta_{n} \rho+\beta_{p} \alpha \rho\right)(d \omega+\Omega \omega+\delta \omega+\mu \omega)}{d(\alpha+d)(d+\Omega+\mu)(d+\delta+\mu)(d+\mu+\omega)}
$$

This measures the average number of new infections generated by a single infectious birds in the poultry.

The steady states are obtained by setting

$$
\frac{d S_{n}}{d t}=\frac{d S_{p}}{d t}=\frac{d E}{d t}=\frac{d I_{p}}{d t}=\frac{d I_{n}}{d t}=\frac{d R}{d t}=0
$$

Thus,

(i) $\left(\rho+c N_{h}\right)-\beta_{n}\left(\boldsymbol{I}_{\boldsymbol{p}}+\boldsymbol{I}_{\boldsymbol{n}}\right) S_{n}-\alpha S_{n}-d S_{n}+\gamma R=0$

(ii) $\alpha S_{n}-\beta_{p}\left(\boldsymbol{I}_{\boldsymbol{p}}+\boldsymbol{I}_{\boldsymbol{n}}\right) S_{p}-d S_{p}=0$

(iii) $\beta_{n}\left(\boldsymbol{I}_{\boldsymbol{p}}+\boldsymbol{I}_{\boldsymbol{n}}\right) S_{n}+\beta_{p}\left(\boldsymbol{I}_{\boldsymbol{p}}+\boldsymbol{I}_{\boldsymbol{n}}\right) S_{p}-\omega \mathrm{E}-(\mu+d) E=0$

(iv) $\omega \mathrm{E}-\Omega I_{p}-(\mu+d) I_{p}=0$

(v) $\Omega I_{p}-\delta I_{n}-(\mu+d) I_{n}=0$

(vi) $\delta I_{n}-(\gamma+d) R=0$

From (i)

(vii) $S_{n}^{*}=\frac{\rho+\gamma R^{*}}{\beta_{n}\left(I_{p}^{*}+I_{n}^{*}\right)+\alpha+d}$

$$
S_{n}^{*}=\frac{(\rho+C N)+\gamma R^{*}}{\beta_{n}\left(I_{p}^{*}+I_{n}^{*}\right)+\alpha+d}, S_{p}^{*}=\frac{\alpha S_{n}^{*}}{\beta_{p}\left(I_{p}^{*}+I_{E}^{*}\right)+d}, \mathrm{E}^{*}=\frac{\left(\rho+C N+\gamma R^{*}\right)\left(\beta_{n}\left(I_{p}^{*}+I_{n}^{*}\right)\right.}{\left(\beta_{n}\left(I_{p}^{*}+I_{n}^{*}\right)+\alpha+d\right)(\omega+\mu+d)}+\frac{\beta_{p}\left(\left(I_{p}^{*}+I_{n}^{*}\right) S_{p}^{*}\right.}{(\omega+\mu+d)}, I_{p}^{*}=\frac{\omega E^{*}}{\Omega+\mu+d}, I_{n}^{*}=\frac{\Omega I_{p}^{*}}{\delta+\mu+d}, R^{*}=\frac{\delta I_{n}^{*}}{\gamma+d}
$$

\subsection{Local Stability of the DFE for NDV Model}

The disease-free equilibrium of the NDV model is given by

$$
\varepsilon_{0}=\left(\mathrm{S}_{\mathrm{n}}^{0}, \mathrm{~S}_{\mathrm{p}}^{0}, 0,0,0,0\right)=\left(\frac{\rho+c N}{\alpha+d}, \frac{\alpha(\rho+c N)}{(\alpha+d) \alpha}, 0,0,0,0\right)
$$

Theorem 3.1: The disease-free steady (equilibrium) state (DFE) for NDV model is locally asymptotically stable if $\mathrm{R}_{0}<1$. Proof: The Jacobian of the NDV model, is given by

$$
J=\left(\begin{array}{cccccc}
c-\beta_{n}\left(I_{p}+I_{n}\right)-\alpha+d & 0 & 0 & c-\beta_{n} S_{n} & c-\beta_{n} S_{n} & \gamma \\
\alpha & -\left(\beta_{n}\left(I_{p}+I_{n}\right)+d\right) & 0 & -\beta_{p} S_{p} & -\beta_{p} S_{p} & 0 \\
\beta_{n}\left(I_{p}+I_{n}\right) & \beta_{p}\left(I_{p}+I_{n}\right) & -(\omega+\mu+d) & \beta_{n} S_{n}+\beta_{p} S_{p} & \beta_{n} S_{n}+\beta_{p} S_{p} & 0 \\
0 & 0 & 0 & -(\delta+\mu+d) & \Omega & 0 \\
0 & 0 & \omega & 0 & -(\Omega+\mu+d) & 0 \\
0 & 0 & 0 & \delta & 0 & -(\gamma+d)
\end{array}\right)
$$

At the DFE, the Jacobian is given by,
From (ii)

(viii) $\alpha S_{n}=\left(\beta_{p}\left(\boldsymbol{I}_{\boldsymbol{p}}+\boldsymbol{I}_{\boldsymbol{n}}\right)+d\right) S_{p}=>S_{p}^{*}=\frac{\alpha S_{n}^{*}}{\beta_{p}\left(I_{p}^{*}+I_{E}^{*}\right)+d}$

From (iii)

(ix) $S_{n}^{*}=\frac{(\omega+\mu+d) E^{*}-\beta_{p}\left(\left(I_{p}^{*}+I_{n}^{*}\right) S_{p}^{*}\right.}{\beta_{n}\left(\left(I_{p}^{*}+I_{n}^{*}\right)\right.}$

From (vii) and (viii)

$$
\begin{gathered}
\frac{\rho+\gamma R^{*}}{\beta_{n}\left(I_{p}^{*}+I_{n}^{*}\right)+\alpha+d}=\frac{(\omega+\mu+d) E^{*}-\beta_{p}\left(\left(I_{p}^{*}+I_{n}^{*}\right) S_{p}^{*}\right.}{\beta_{n}\left(\left(I_{p}^{*}+I_{n}^{*}\right)\right.} \\
\mathrm{E}^{*}=\frac{\left(\rho+\gamma R^{*}\right)\left(\beta_{n}\left(I_{p}^{*}+I_{n}^{*}\right)\right.}{\left(\beta_{n}\left(I_{p}^{*}+I_{n}^{*}\right)+\alpha+d\right)(\omega+\mu+d)}+\frac{\beta_{p}\left(\left(I_{p}^{*}+I_{n}^{*}\right) S_{p}^{*}\right.}{(\omega+\mu+d)}
\end{gathered}
$$

From (iv)

$$
\omega \mathrm{E}=\Omega I_{p}+(\mu+d) I_{p}=>I_{p}^{*}=\frac{\omega E^{*}}{\Omega+\mu+d}
$$

From (v)

$$
\Omega I_{p}=\delta I_{n}+(\mu+d) I_{n}=>I_{n}^{*}=\frac{\Omega I_{p}^{*}}{\delta+\mu+d}
$$

$$
\delta I_{n}=(\gamma+d) R=>R^{*}=\frac{\delta I_{n}^{*}}{\gamma+d}
$$

The steady states are; 


$$
J_{D F E}=\left(\begin{array}{cccccc}
c-\alpha+d & 0 & 0 & c-\frac{\beta_{n} \rho}{\alpha+d} & c-\frac{\beta_{n} \rho}{\alpha+d} & \gamma \\
\alpha & -d & 0 & -\frac{\beta_{p} \alpha \rho}{d(\alpha+d)} & -\frac{\beta_{p} \alpha \rho}{d(\alpha+d)} & 0 \\
0 & 0 & -(\omega+\mu+d) & \frac{\beta_{n} \rho}{\alpha+d}+\frac{\beta_{p} \alpha \rho}{d(\alpha+d)} & \frac{\beta_{n} \rho}{\alpha+d}+\frac{\beta_{p} \alpha \rho}{d(\alpha+d)} & 0 \\
0 & 0 & 0 & -(\delta+\mu+d) & \Omega & 0 \\
0 & 0 & \omega & 0 & -(\Omega+\mu+d) & 0 \\
0 & 0 & 0 & \delta & 0 & -(\gamma+d)
\end{array}\right)
$$

Considering the determinant

$$
\left|J_{D F E}-\lambda\right|=\left|\begin{array}{cccccc}
c-\alpha+d-\lambda & 0 & 0 & c-\frac{\beta_{n} \rho}{\alpha+d} & c-\frac{\beta_{n} \rho}{\alpha+d} & \gamma \\
\alpha & -d-\lambda & 0 & -\frac{\beta_{p} \alpha \rho}{d(\alpha+d)} & -\frac{\beta_{p} \alpha \rho}{d(\alpha+d)} & 0 \\
0 & 0 & -(\omega+\mu+d)-\lambda & \frac{\beta_{n} \rho}{\alpha+d}+\frac{\beta_{p} \alpha \rho}{d(\alpha+d)} & \frac{\beta_{n} \rho}{\alpha+d}+\frac{\beta_{p} \alpha \rho}{d(\alpha+d)} & 0 \\
0 & 0 & 0 & -(\delta+\mu+d)-\lambda & \Omega & 0 \\
0 & 0 & \omega & 0 & -(\Omega+\mu+d)-\lambda & 0 \\
0 & 0 & 0 & \delta & 0 & -(\gamma+d)-\lambda
\end{array}\right|
$$

Evaluating the determinant gives,

where

$$
\begin{gathered}
(-\mathrm{d}-\lambda)\left(\mathrm{k}_{1}-\lambda\right)(-\mathrm{d}-\gamma-\lambda)\left[-\left(\mathrm{k}_{2}-\lambda\right)\left(\mathrm{k}_{3}-\lambda\right)\left(\mathrm{k}_{4}-\lambda\right)+\beta_{n} \mathrm{k}_{1}+\right. \\
\left.\beta_{p} \mathrm{k}_{1}+\lambda\left(\beta_{n}+\beta_{p}\right)\right]=0
\end{gathered}
$$

Where

$\mathrm{k}_{1}=(d \omega+\Omega \omega+\delta \omega+\mu \omega), \mathrm{k}_{2}=d+\Omega+\mu, \mathrm{k}_{3}=d+\delta+\mu$,

$$
\mathrm{k}_{4}=d+\mu+\omega
$$

From the above equation, three of the eigen values are

$$
\lambda_{1}=-\mathrm{d}<0, \lambda_{2}=-\mathrm{k}_{1}<0, \lambda_{3}=-\mathrm{d}-\gamma<0
$$

The remaining eigenvalues can be resolved from

$$
\begin{gathered}
{\left[-(\mathrm{k} 2-\lambda)(\mathrm{k} 3-\lambda)(\mathrm{k} 4-\lambda)+\beta_{n} \mathrm{k} 1+\beta_{p} \mathrm{k} 1+\lambda\left(\beta_{n}+\beta_{p}\right)\right]=0} \\
\lambda^{3}+\left(k_{3}+k_{4}+k_{2}\right) \lambda^{2} \\
+\left(k_{2} k_{3}+k_{2} k_{4}+k_{3} k_{4}-\beta_{n}-\beta_{p}\right) \lambda+k_{2} k_{3}-k_{1}\left(\beta_{n}+\beta_{p}\right)=0 \\
\lambda^{3}+\left(k_{3}+k_{4}+k_{2}\right) \lambda^{2}
\end{gathered}
$$$$
+\left(k_{2} k_{3}+k_{2} k_{4}+k_{3} k_{4}-\beta_{n}-\beta_{p}\right) \lambda+k_{2} k_{3} k_{4}\left(1-R_{0}\right)=0
$$$$
\varepsilon_{1}=\left(0,0, \mathrm{E}^{*}, \mathrm{I}_{\mathrm{p}}{ }^{*}, \mathrm{I}_{\mathrm{n}}{ }^{*}\right)=\left(0,0, \frac{\left(\rho+c N+\gamma R^{*}\right)\left(\beta_{n}\left(I_{p}^{*}+I_{n}^{*}\right)\right.}{\left(\beta_{n}\left(I_{p}^{*}+I_{n}^{*}\right)+\alpha+d\right)(\omega+\mu+d)}+\frac{\beta_{p}\left(\left(I_{p}^{*}+I_{n}^{*}\right)\right)_{p}^{*}}{(\omega+\mu+d)}, \frac{\omega E^{*}}{\Omega+\mu+d}, \frac{\Omega I_{p}^{*}}{\delta+\mu+d}\right)
$$

Theorem 3.2: The endemic steady (equilibrium) state of the NDV is locally asymptotically stable when $R_{0}>1$ if

$$
\beta_{n}>\frac{c-\alpha-d}{I_{p}+I_{n}}
$$

Proof: The Jacobian at the endemic equilibrium gives

$$
R_{0}=\frac{k_{1}\left(\beta_{n}+\beta_{p}\right)}{k_{2} k_{3} k_{4}}
$$

By Rourth-Horwith condition: The polynomial has negative real roots if $a_{0}>0, a_{1}>0, a_{3}>0$ and $a_{1} a_{2}>a_{0} a_{3}$. From the equation; $\mathrm{a}_{0}=1>0, \mathrm{a}_{1}=\left(k_{3}+k_{4}+k_{2}\right)>0, \mathrm{a}_{3}=k_{2} k_{3} k_{4}(1-$ $\left.R_{0}\right)>0$ if and only if $R_{0}<1$ and $\mathrm{a}_{1} \mathrm{a}_{2}>\mathrm{a}_{0} \mathrm{a}_{3}$ is satisfied.

Hence we conclude that the DFE is locally asymptotically stable whenever $\mathrm{R}_{0}<1$.

The epidemiological implication of the result is that the spread of Newcastle disease virus can be effectively controlled in the poultry when the basic reproduction number $\mathrm{R}_{0}<1$, provided the initial population contains a small influx of infectious birds.

\subsection{Local Stability of the EE for NDV Model}

In a pure endemic state, we have that $S_{n}=S_{p}=R=0$. Hence, the endemic equilibrium becomes, 


$$
\begin{aligned}
& J_{E E}=\left(\begin{array}{cc}
c-\beta_{n}\left(I_{p}+I_{n}\right)-\alpha+d & 0 \\
\alpha & -\left(\beta_{n}\left(I_{p}+I_{n}\right)+d\right) \\
\beta_{n}\left(I_{p}+I_{n}\right) & \beta_{p}\left(I_{p}+I_{n}\right) \\
0 & 0 \\
0 & 0 \\
0 & 0
\end{array}\right. \\
& \beta_{n}\left(I_{p}+I_{n}\right)-\alpha+d, \lambda_{2}=-\left(\beta_{n}\left(I_{p}+I_{n}\right)+d\right), \lambda_{3}= \\
& +d), \lambda_{4}=-(\delta+\mu+d), \lambda_{5}=-(\Omega+\mu+d) \text { and } \lambda_{6}= \\
& -(\gamma+d)
\end{aligned}
$$

The system will be stable if $\lambda_{1}<0$, that is

$$
c-\beta_{n}\left(I_{p}+I_{n}\right)-\alpha+d<0=>\beta_{n}>\frac{c-\alpha-d}{I_{p}+I_{n}}
$$

This means that if the contact rate for non-productive infectious birds $\left(\beta_{n}\right)$ exceeds the quantity $\frac{c-\alpha-d}{I_{p}+I_{n}}$, then the

$$
\begin{aligned}
& d_{1}=(d \rho+\alpha \rho)(d \omega+\Omega \omega+\delta \omega+\mu \omega), \\
& d_{2}=d(\alpha+d)(d+\Omega+\mu)(d+\mu+\omega), \\
& d_{3}=d(\alpha+d)(d+\mu+\omega)(d+\delta+\mu+\Omega)
\end{aligned}
$$

with Lyaponuv derivative given by

$$
\begin{aligned}
\dot{L} & =d_{1} \dot{E}+d_{2} \dot{I}_{p}+d_{3} I_{n} \\
& =d_{1}\left[\beta_{n}\left(I_{p}+I_{n}\right) S_{n}+\beta_{p}\left(I_{p}+I_{n}\right) S_{p}-\omega E-(\mu+d) E\right]+d_{2}\left[\omega E-\Omega I_{p}-(\mu+d) I_{p}\right]+d_{3}\left[\Omega I_{p}-\delta I_{n}-(\mu+d) I_{n}\right] \\
& =\beta\left(S_{n}+S_{p}\right)\left(I_{p}+I_{n}\right)(d \rho+\alpha \rho)(d \omega+\Omega \omega+\delta \omega+\mu \omega)-(d+\Omega+\mu)(d+\mu+\omega)(d+\delta+\mu)\left(I_{p}+I_{n}\right) \\
& =\left(I_{p}+I_{n}\right)\left[\frac{\beta\left(S_{n}+S_{p}\right)\left(I_{p}+I_{n}\right)(d \rho+\alpha \rho)(d \omega+\Omega \omega+\delta \omega+\mu \omega)}{d(\alpha+d)(d+\Omega+\mu)(d+\mu+\omega)(d+\delta+\mu)}-1\right]
\end{aligned}
$$

Since

$$
S_{n} \leq S_{n}^{*} \text { and } S_{p} \leq S_{p}^{*}
$$

in the domain that forms the invariant set, it then follows that

$$
\dot{L} \leq\left(I_{p}+I_{n}\right)\left[\frac{\beta(d \rho+\alpha \rho)(d \omega+\Omega \omega+\delta \omega+\mu \omega)}{(d+\Omega+\mu)(d+\mu+\omega)(d+\delta+\mu)}-1\right]=\left(I_{p}+I_{n}\right)\left(R_{0}-1\right)
$$

Clearly $\dot{L} \leq 0$ if $R_{0} \leq 1$ Equality is achieved at $\mathrm{I}_{\mathrm{p}}=\mathrm{I}_{\mathrm{n}}=0$, Hence by Lassalle's invariance principle, every solution in the invariant set for which $\mathrm{t} \geq 0$ approaches the DFE that is, $\varepsilon_{0}=\left(\mathrm{S}_{\mathrm{n}}{ }^{0}, \mathrm{~S}_{\mathrm{p}}{ }^{0}, 0,0,0,0\right)$. Hence, the DFE is globally asymptotically stable when $\mathrm{R}_{0}<1$.

\subsection{Global Stability of the EE for NDV Model}

The global asymptotic stability analysis of the endemic equilibrium for the NDV model is obtained for the special case with $\mu=0$ and without exogenous re infection. To achieve this, we use the nonlinear Lyapunov function of Goh-Volterra type. The new model equation with $\mu=0, \gamma=0$ and $\rho+c N_{h}=\rho$ becomes 


$$
\begin{gathered}
\frac{d S_{n}}{d t}=\rho-\beta_{n}\left(\boldsymbol{I}_{\boldsymbol{p}}+\boldsymbol{I}_{\boldsymbol{n}}\right) S_{n}-k_{1} S_{n} \\
\frac{d S_{p}}{d t}=\alpha S_{n}-\beta_{p}\left(\boldsymbol{I}_{p}+\boldsymbol{I}_{\boldsymbol{n}}\right) S_{p}-d S_{p} \\
\frac{d E}{d t}=\beta_{n}\left(\boldsymbol{I}_{p}+\boldsymbol{I}_{\boldsymbol{n}}\right) S_{n}+\beta_{p}\left(\boldsymbol{I}_{p}+\boldsymbol{I}_{\boldsymbol{n}}\right) S_{p}-\mathrm{K}_{2} \mathrm{E} \\
\frac{d I_{p}}{d t}=\omega \mathrm{E}-k_{3} I_{p} \\
\frac{d I_{p}}{d t}=\Omega I_{p}-k_{4} I_{n}
\end{gathered}
$$

where

$$
\mathrm{k}_{1}=\alpha+d, \mathrm{k}_{2}=\omega+d, \mathrm{k}_{3}=\Omega+d, \mathrm{k}_{4}=\delta+d
$$

Theorem 3.4: Theendemic equilibrium of the model is globally asymptotically stable if $\mathrm{R}_{0}>1$

Proof: Consider the following nonlinear Lyaponuv function

$$
L=S_{n}-S_{n}^{*}-S_{n}^{*} \ln \frac{S_{n}}{S_{n}^{*}}+S_{p}-S_{p}^{*}-S_{p}^{*} \ln \frac{S_{p}}{S_{p}^{*}}+E-E^{*}-E^{*} \ln \frac{E}{E^{*}}+A\left(I_{p}-I_{p}^{*}-I_{p}^{*} \ln \frac{I_{p}}{I_{p}^{*}}\right)+B\left(I_{n}-I_{n}^{*}-I_{n}^{*} \ln \frac{I_{n}}{I_{n}^{*}}\right)
$$

With Lyaponuv derivative given as

$$
\begin{aligned}
& \dot{L}=\left(\dot{S}_{n}-\frac{S_{n}^{*}}{S_{n}} \dot{S}_{n}\right)+\left(\dot{S}_{p}-\frac{S_{p}^{*}}{S_{p}} \dot{S}_{p}\right)+\left(\dot{E}-\frac{E^{*}}{E} \dot{E}\right)+A\left(\dot{I}_{p}-\frac{I_{p}^{*}}{I_{p}} \dot{I}_{p}\right)+B\left(\dot{I}_{n}-\frac{I_{n}^{*}}{I_{n}} \dot{I}_{n}\right) \\
& \text { where } A=\left(\frac{\beta_{n} S_{n}^{*}+\beta_{p} S_{p}^{*}}{\Omega+d}\right)+\left(\frac{\left(\beta_{n} S_{n}^{*}+\beta_{p} S_{p}^{*}\right) \Omega}{(\Omega+d)(\delta+d)}\right) \text { and } B=\left(\frac{\beta_{n} S_{n}^{*}+\beta_{p} S_{p}^{*}}{\delta+d}\right)
\end{aligned}
$$

Putting the appropriate equations from model (3.1) we have

$$
\begin{aligned}
& \dot{L}=\left[\rho-\beta_{n}\left(\boldsymbol{I}_{\boldsymbol{p}}+\boldsymbol{I}_{\boldsymbol{n}}\right) S_{n}-\alpha S_{n}-d S_{n}-\frac{S_{n}^{*}}{S_{n}}\left(\rho-\beta_{n}\left(\boldsymbol{I}_{\boldsymbol{p}}+\boldsymbol{I}_{\boldsymbol{n}}\right) S_{n}-\alpha S_{n}-d S_{n}\right)\right]+ \\
& {\left[\alpha S_{n}-\beta_{p}\left(\boldsymbol{I}_{\boldsymbol{p}}+\boldsymbol{I}_{\boldsymbol{n}}\right) S_{p}-d S_{p}-\frac{S_{p}^{*}}{S_{p}}\left(\alpha S_{n}-\beta_{p}\left(\boldsymbol{I}_{\boldsymbol{p}}+\boldsymbol{I}_{\boldsymbol{n}}\right) S_{p}-d S_{p}\right)\right]+} \\
& \quad\left[\beta_{n}\left(\boldsymbol{I}_{\boldsymbol{p}}+\boldsymbol{I}_{\boldsymbol{n}}\right) S_{n}+\beta_{p}\left(\boldsymbol{I}_{\boldsymbol{p}}+\boldsymbol{I}_{\boldsymbol{n}}\right) S_{p}-\Upsilon(\omega+d) E-\frac{E^{*}}{E}\left(\beta_{n}\left(\boldsymbol{I}_{\boldsymbol{p}}+\boldsymbol{I}_{\boldsymbol{n}}\right) S_{n}+\beta_{p}\left(\boldsymbol{I}_{\boldsymbol{p}}+\boldsymbol{I}_{\boldsymbol{n}}\right) S_{p}-\Upsilon(\omega+d) E\right)\right] \\
& \quad+\left(\beta_{n} S_{n}^{*}+\beta_{p} S_{p}^{*}\right)\left(\frac{1}{\Omega+d}+\frac{\Omega}{(\Omega+d)(\boldsymbol{\delta}+d)}\right)\left[\omega \mathrm{E}-(\Omega+d) I_{p}-\frac{I_{p}^{*}}{I_{p}}\left(\omega \mathrm{E}-(\Omega+d) I_{p}\right)\right]+ \\
& \quad\left(\frac{\beta_{n} S_{n}^{*}+\beta_{p} S_{p}^{*}}{\delta+d}\right)\left[\Omega I_{p}-(\delta+d) I_{n}-\frac{I_{n}^{*}}{I_{n}}\left(\Omega I_{p}-(\delta+d) I_{n}\right)\right]
\end{aligned}
$$

From (2), at steady states

$$
\begin{gathered}
\rho=\beta_{n}\left(\boldsymbol{I}_{p}^{*}+\boldsymbol{I}_{n}^{*}\right) \mathrm{S}_{n}^{*}+k_{1} S_{n}^{*} \\
(\omega+d)=\frac{\beta_{n}\left(I_{p}^{*}+I_{n}^{*}\right) S_{n}^{*}+\beta_{p}\left(I_{p}^{*}+I_{n}^{*}\right) S_{p}^{*}}{E^{*}} \\
\omega=\frac{(\Omega+\mathrm{d}) I_{p}^{*}}{E^{*}}
\end{gathered}
$$


and

$$
(\delta+d)=\frac{\Omega I_{p}^{*}}{I_{n}^{*}}
$$

Substituting $\rho$ at steady state, gives

$$
\begin{aligned}
& \dot{L}=\left[\beta_{n}\left(\boldsymbol{I}_{p}^{*}+\boldsymbol{I}_{n}^{*}\right) \mathrm{S}_{n}^{*}+\alpha S_{n}^{*}+d S_{n}^{*}-\beta_{n}\left(\boldsymbol{I}_{\boldsymbol{p}}+\boldsymbol{I}_{\boldsymbol{n}}\right) S_{n}-\alpha S_{n}-d S_{n}-\frac{S_{n}^{*}}{S_{n}}\left(\left(\beta_{n}\left(\boldsymbol{I}_{p}^{*}+\boldsymbol{I}_{n}^{*}\right) \mathrm{S}_{n}^{*}+\alpha S_{n}^{*}+\mathrm{d} S_{n}^{*}\right)-\beta_{n}\left(\boldsymbol{I}_{\boldsymbol{p}}+\boldsymbol{I}_{\boldsymbol{n}}\right) S_{n}-k_{1} S_{n}\right)\right]+ \\
& {\left[\alpha S_{n}-\beta_{p}\left(\boldsymbol{I}_{\boldsymbol{p}}+\boldsymbol{I}_{\boldsymbol{n}}\right) S_{p}-d S_{p}-\frac{S_{p}^{*}}{S_{p}}\left(\alpha S_{n}-\beta_{p}\left(\boldsymbol{I}_{\boldsymbol{p}}+\boldsymbol{I}_{\boldsymbol{n}}\right) S_{p}-d S_{p}\right)\right]+} \\
& {\left[\beta_{n}\left(\boldsymbol{I}_{\boldsymbol{p}}+\boldsymbol{I}_{\boldsymbol{n}}\right) S_{n}+\beta_{p}\left(\boldsymbol{I}_{\boldsymbol{p}}+\boldsymbol{I}_{\boldsymbol{n}}\right) S_{p}-\gamma(\boldsymbol{\omega}+d) E-\frac{E^{*}}{E}\left(\beta_{n}\left(\boldsymbol{I}_{\boldsymbol{p}}+\boldsymbol{I}_{\boldsymbol{n}}\right) S_{n}+\beta_{p}\left(\boldsymbol{I}_{\boldsymbol{p}}+\boldsymbol{I}_{\boldsymbol{n}}\right) S_{p}-\gamma(\omega+d) E\right)\right]} \\
& +\left(\beta_{n} S_{n}^{*}+\beta_{p} S_{p}^{*}\right)\left(\frac{1}{\Omega+d}+\frac{\Omega}{(\Omega+d)(\delta+d)}\right)\left[\omega \mathrm{E}-(\Omega+d) I_{p}-\frac{I_{p}^{*}}{I_{p}}\left(\omega \mathrm{E}-(\Omega+d) I_{p}\right)\right]+ \\
& \left(\frac{\beta_{n} S_{n}^{*}+\beta_{p} S_{p}^{*}}{\delta+d}\right)\left[\Omega I_{p}-(\delta+d) I_{n}-\frac{I_{n}^{*}}{I_{n}}\left(\Omega I_{p}-(\delta+d) I_{n}\right)\right]
\end{aligned}
$$

Substituting the values of $(\Omega+d), \omega$ and $(\delta+d)$ at steady states, we have,

$$
\begin{aligned}
& \dot{L}=\beta_{n}\left(I_{p}^{*}+I_{n}^{*}\right) \mathrm{S}_{n}^{*}+2\left(\beta_{p} S_{p}^{*}\left(I_{p}^{*}+I_{n}^{*}\right)+\mathrm{dS}_{p}^{*}\right)+\mathrm{dS}_{n}^{*}-d S_{n}-\frac{S_{n}^{* 2}}{S_{n}} \beta_{n}\left(I_{p}^{*}+\boldsymbol{I}_{n}^{*}\right)-\left(\beta_{p} S_{p}^{*}\left(I_{p}^{*}+I_{n}^{*}\right)+\mathrm{d} S_{p}^{*}\right) \frac{S_{n}^{*}}{S_{n}}-\frac{d S_{n}^{* 2}}{S_{n}}+d S_{n}^{*}-d S_{p} \\
& -\left(\beta_{p} S_{p}^{*}\left(I_{p}^{*}+I_{n}^{*}\right)+\mathrm{dS}_{p}^{*}\right) \frac{S_{n} S_{p}^{*}}{S_{n}^{*} S_{p}}+d S_{p}^{*}-\frac{E^{*}}{E} \beta_{n}\left(\boldsymbol{I}_{p}+\boldsymbol{I}_{n}\right) S_{n}-\frac{E^{*}}{E} \beta_{p}\left(\boldsymbol{I}_{\boldsymbol{p}}+\boldsymbol{I}_{n}\right) S_{p}+\beta_{n} S_{n}^{*}\left(I_{p}^{*}+I_{n}^{*}\right)+\beta_{p} S_{p}^{*}\left(I_{p}^{*}+I_{n}^{*}\right) \\
& -\left(\beta_{n} S_{n}^{*}+\beta_{p} S_{p}^{*}\right)\left(\frac{I_{p}^{* 2} E}{I_{p} E^{*}}+\frac{I_{p}^{*} I_{n}^{*} E}{I_{p} E^{*}}\right)+\left(\beta_{n} S_{n}^{*}+\beta_{p} S_{p}^{*}\right)\left(I_{p}^{*}+I_{n}^{*}\right)-\left(\beta_{n} S_{n}^{*}+\beta_{p} S_{p}^{*}\right) \frac{I_{n}^{* 2} I_{p}}{I_{n} I_{p}^{*}}+\left(\beta_{n} S_{n}^{*}+\beta_{p} S_{p}^{*}\right) I_{n}^{*}
\end{aligned}
$$

Collecting terms with $d S_{n}^{*}, d S_{p}^{*}, \beta_{n} S_{n}^{*} I_{n}^{*}, \beta_{n} S_{n}^{*} I_{p}^{*}, \beta_{p} S_{p}^{*} I_{p}^{*}$ and $\beta_{p} S_{p}^{*} I_{n}^{*}$ gives,

$$
\begin{aligned}
& \dot{L}=d S_{n}^{*}\left[2-\frac{S_{n}}{S_{n}^{*}}-\frac{S_{n}^{*}}{S_{n}}\right]+d S_{p}^{*}\left[3-\frac{S_{n}^{*}}{S_{n}}-\frac{S_{p}}{S_{p}^{*}}-\frac{S_{n} S_{p}^{*}}{S_{n}^{*} S_{p}}\right]+\beta_{n} S_{n}^{*} I_{n}^{*}\left[4-\frac{S_{n}^{*}}{S_{n}}-\frac{S_{n} E^{*} I_{n}}{S_{n}^{*} E I_{n}^{*}}-\frac{I_{p}^{*} E}{I_{p} E^{*}}-\frac{I_{n}^{*} I_{p}}{I_{n} I_{p}^{*}}\right] \\
& +\beta_{p} S_{p}^{*} I_{p}^{*}\left[4-\frac{S_{n}^{*}}{S_{n}}-\frac{S_{n} S_{p}^{*}}{S_{n}^{*} S_{p}}-\frac{S_{p} I_{p} E^{*}}{S_{p}^{*} I_{p}^{*} E}-\frac{I_{p}^{*} E}{I_{p} E^{*}}\right]+\beta_{n} S_{n}^{*} I_{p}^{*}\left[3-\frac{S_{n}^{*}}{S_{n}}-\frac{S_{n} E^{*} I_{P}}{S_{n}^{*} E I_{p}^{*}}-\frac{I_{p}^{*} E}{I_{p} E^{*}}\right] \\
& +\beta_{p} S_{p}^{*} I_{n}^{*}\left[5-\frac{S_{n}^{*}}{S_{n}}-\frac{S_{n} S_{p}^{*}}{S_{n}^{*} S_{p}}-\frac{S_{p} I_{n} E^{*}}{S_{p}^{*} I_{n}^{*} E}-\frac{I_{p}^{*} E}{I_{p} E^{*}}-\frac{I_{n}^{*} I_{p}}{I_{n} I_{p}^{*}}\right]
\end{aligned}
$$

Finally since the arithmetic mean exceeds the geometric mean, that is,

$$
\mathrm{AM}=\frac{a_{1}+a_{2}+a_{3}+\cdots+a_{n}}{n} \geq \sqrt[n]{a_{1} a_{2} a_{3} \ldots a_{n}}=G M
$$

It follows that,

$$
\begin{gathered}
d S_{n}^{*}\left[2-\frac{S_{n}}{S_{n}^{*}}-\frac{S_{n}^{*}}{S_{n}}\right] \leq 0, d S_{p}^{*}\left[3-\frac{S_{n}^{*}}{S_{n}}-\frac{S_{p}}{S_{p}^{*}}-\frac{S_{n} S_{p}^{*}}{S_{n}^{*} S_{p}}\right] \leq 0, \beta_{n} S_{n}^{*} I_{n}^{*}\left[4-\frac{S_{n}^{*}}{S_{n}}-\frac{S_{n} E^{*} I_{n}}{S_{n}^{*} E I_{n}^{*}}-\frac{I_{p}^{*} E}{I_{p} E^{*}}-\frac{I_{n}^{*} I_{p}}{I_{n} I_{p}^{*}}\right] \leq 0 \\
\beta_{p} S_{p}^{*} I_{p}^{*}\left[4-\frac{S_{n}^{*}}{S_{n}}-\frac{S_{n} S_{p}^{*}}{S_{n}^{*} S_{p}}-\frac{S_{p} I_{p} E^{*}}{S_{p}^{*} I_{p}^{*} E}-\frac{I_{p}^{*} E}{I_{p} E^{*}}\right] \leq 0, \beta_{n} S_{n}^{*} I_{p}^{*}\left[3-\frac{S_{n}^{*}}{S_{n}}-\frac{S_{n} E^{*} I_{P}}{S_{n}^{*} E I_{p}^{*}}-\frac{I_{p}^{*} E}{I_{p} E^{*}}\right] \leq 0,
\end{gathered}
$$




$$
\beta_{p} S_{p}^{*} I_{n}^{*}\left[5-\frac{S_{n}^{*}}{S_{n}}-\frac{S_{n} S_{p}^{*}}{S_{n}^{*} S_{p}}-\frac{S_{p} I_{n} E^{*}}{S_{p}^{*} I_{n}^{*} E}-\frac{I_{p}^{*} E}{I_{p} E^{*}}-\frac{I_{n}^{*} I_{p}}{I_{n} I_{p}^{*}}\right] \leq 0
$$

That is,

$$
\begin{gathered}
2-\frac{S_{n}}{S_{n}^{*}}-\frac{S_{n}^{*}}{S_{n}}=2-\left(\frac{S_{n}}{S_{n}^{*}}+\frac{S_{n}^{*}}{S_{n}}\right) \\
A \cdot M=\frac{\left(\frac{S_{n}}{S_{n}^{*}}+\frac{S_{n}^{*}}{S_{n}}\right)}{2}, G \cdot M=\sqrt[2]{\frac{S_{n}}{S_{n}^{*}} \cdot \frac{S_{n}^{*}}{S_{n}}}=1
\end{gathered}
$$

Since G.M-A.M $\leq$, hence

$$
1-\left(\frac{S_{n}}{S_{n}^{*}}+\frac{S_{n}^{*}}{S_{n}}\right) \leq 0
$$

Thus

$$
2-\frac{S_{n}}{S_{n}^{*}}-\frac{S_{n}^{*}}{S_{n}} \leq 0
$$

Using similar approach we can also show that

$$
\begin{gathered}
3-\frac{S_{n}^{*}}{S_{n}}-\frac{S_{p}}{S_{p}^{*}}-\frac{S_{n} S_{p}^{*}}{S_{n}^{*} S_{p}} \leq 0,4-\frac{S_{n}^{*}}{S_{n}}-\frac{S_{n} E^{*} I_{n}}{S_{n}^{*} E I_{n}^{*}}-\frac{I_{p}^{*} E}{I_{p} E^{*}}-\frac{I_{n}^{*} I_{p}}{I_{n} I_{p}^{*}} \leq 0, \\
4-\frac{S_{n}^{*}}{S_{n}}-\frac{S_{n} S_{p}^{*}}{S_{n}^{*} S_{p}}-\frac{S_{p} I_{p} E^{*}}{S_{p}^{*} I_{p}^{*} E}-\frac{I_{p}^{*} E}{I_{p} E^{*}} \leq 03-\frac{S_{n}^{*}}{S_{n}}-\frac{S_{n} E^{*} I_{P}}{S_{n}^{*} E I_{p}^{*}}-\frac{I_{p}^{*} E}{I_{p} E^{*}} \leq 0
\end{gathered}
$$

and

$$
5-\frac{S_{n}^{*}}{S_{n}}-\frac{S_{n} S_{p}^{*}}{S_{n}^{*} S_{p}}-\frac{S_{p} I_{n} E^{*}}{S_{p}^{*} I_{n}^{*} E}-\frac{I_{p}^{*} E}{I_{p} E^{*}}-\frac{I_{n}^{*} I_{p}}{I_{n} I_{p}^{*}} \leq 0
$$

Furthermore, since all the model parameters are nonnegative, it follows that $\dot{L} \leq 0$ for $\mathrm{R}_{0}>1$ with $\dot{L}=0$ if and only if $\mathrm{S}_{\mathrm{n}}=\mathrm{S}_{\mathrm{n}}{ }^{*}, \mathrm{~S}_{\mathrm{p}}=\mathrm{S}_{\mathrm{p}}{ }^{*}, \mathrm{E}=\mathrm{E}^{*}, \mathrm{I}_{\mathrm{p}}=\mathrm{I}_{\mathrm{p}}{ }^{*}, \mathrm{I}_{\mathrm{n}}=\mathrm{I}_{\mathrm{n}}{ }^{*}$. Hence the largest invariant subset of the set where $\dot{L}=0$ is the singleton $\left\{\left(\mathrm{S}_{\mathrm{n}}, \mathrm{S}_{\mathrm{p}}\right.\right.$, $\left.\left.\mathrm{E}, \mathrm{I}_{\mathrm{p}}, \mathrm{I}_{\mathrm{n}}, \mathrm{R}\right)=\left(\mathrm{S}_{\mathrm{n}}{ }^{*}, \mathrm{~S}_{\mathrm{p}}{ }^{*}, \mathrm{E}^{*}, \mathrm{I}_{\mathrm{p}}{ }^{*}, \mathrm{I}_{\mathrm{n}}{ }^{*}\right)\right\}$. By Lasalle's invariance principle, it follows that every solution in $\mathrm{D}$ approaches the endemic equilibrium $\varepsilon_{1}$ for $\mathrm{R}_{0}>1$ as $\mathrm{t} \rightarrow \infty$.

The epidemiological implication of the above result is that Newcastle disease will remain endemic in the poultry for $\mathrm{R}_{0}>1$, whether the initial population contains a sufficient number of infected birds or not.

\subsection{Sensitivity Analysis on $\boldsymbol{R}_{0}$}

To determine the parameters most responsible for the transmission and spread of Newcastle disease, a sensitivity analysis is carried out. The normalised forward sensitivity index of $\mathrm{R}_{0}$ that depends differentiably on a parameter $\mathrm{p}$ is defined by

$$
r_{p}^{R_{0}}=\left(\frac{\partial R_{0}}{\partial p}\right)\left(\frac{p}{R_{0}}\right)
$$

In particular, sensitivity indices of the basic reproduction number, $\mathrm{R}_{0}$, with respect to the model parameters are computed as follows:

$$
\begin{gathered}
\left(\frac{\partial R_{0}}{\partial \beta_{n}}\right)\left(\frac{\beta_{n}}{R_{0}}\right)=\frac{d \beta_{n}}{d \beta_{n}+\alpha \beta_{p}} \\
\left(\frac{\partial R_{0}}{\partial \beta_{p}}\right)\left(\frac{\beta_{p}}{R_{0}}\right)=\frac{\alpha \beta_{p}}{d \beta_{n}+\alpha \beta_{p}} \\
\left(\frac{\partial R_{0}}{\partial \rho}\right)\left(\frac{\rho}{R_{0}}\right)=1 \\
\left(\frac{\partial R_{0}}{\partial \alpha}\right)\left(\frac{\alpha}{R_{0}}\right)=\frac{d \alpha_{\beta}}{(\alpha+d)\left(d \beta_{n}+\alpha \beta_{p}\right)} \\
\left(\frac{\partial R_{0}}{\partial \Omega}\right)\left(\frac{\Omega}{R_{0}}\right)=\frac{\Omega \delta \omega}{(d \omega+\Omega \omega+\delta \omega+\mu \omega)(d+\Omega+\mu)} \\
\left(\frac{\partial R_{0}}{\partial \omega}\right)\left(\frac{\omega}{R_{0}}\right)=\frac{d+\mu}{(d+\omega+\mu)}
\end{gathered}
$$




$$
\begin{gathered}
\left(\frac{\partial R_{0}}{\partial \delta}\right)\left(\frac{\delta}{R_{0}}\right)=-\frac{-\delta \Omega \omega}{(d \omega+\Omega \omega+\delta \omega+\mu \omega)(d+\delta+\mu)} \\
\left(\frac{\partial R_{0}}{\partial \mu}\right)\left(\frac{\mu}{R_{0}}\right)=-\frac{k_{1}}{(d \omega+\Omega \omega+\delta \omega+\mu \omega)(d+\Omega+\mu)(d+\delta+\mu)(d+\omega+\mu)} \\
\left(\frac{\partial R_{0}}{\partial d}\right)\left(\frac{d}{R_{0}}\right)=-\frac{k_{2}}{(d \omega+\Omega \omega+\delta \omega+\mu \omega)(d+\Omega+\mu)(d+\delta+\mu)(d+\omega+\mu)}
\end{gathered}
$$

The sensitivity index of the basic reproduction number to the model parameters indicates that an increase (or decrease) in the following values; $\beta_{n}, \beta_{p}, \rho, \alpha, \Omega$ and $\omega$ will lead to an increase (or decrease) in the basic reproduction number. On the other hand, an increase (or decrease) in the death rates $(\mathrm{d}, \mu)$ and recovery rate $(\delta)$ leads to a corresponding decrease (or increase) in the basic reproduction number of the disease.

\subsection{Formulation of the Optimal Control Problem for NDV}

Given the initial population size of all the five classes of model (2), our goal is to find the best control strategy that would minimize the number of birds that die as a result of the disease, thereby increasing the number of productive birds and at the same time minimizing the cost of the strategy. The control strategies $v_{1}(t), v_{2}(t)$ and $v_{3}(t)$ represents the efficiency of the vaccine therapy in preventing new infection, the efficiency of drug in inhibiting the virus strain and effort on infected birds to increase the number of recovered birds respectively.
Thus we seek to minimize the objective functional

$$
\mathrm{J}(\mathrm{u}, \mathrm{v})=\int_{0}^{T}\left(B E+C I_{n}+D I_{p}+\frac{A_{1}}{2} v_{1}^{2}+\frac{A_{2}}{2} v_{2}^{2}+\frac{A_{3}}{2} v_{3}^{2}\right)
$$

with $\mathrm{B}>0, \mathrm{C}>0, \mathrm{D}>0, \mathrm{~A}_{1}>0, \mathrm{~A}_{2}>0, \mathrm{~A}_{3}>0$. Here we want to minimize the number of infectious birds while keeping the cost of vaccination and treatment / drug low. The quadratic form represents giving too much of vaccine to the birds which often leads to waste. The terms $\mathrm{CI}_{n}$ and $\mathrm{DI}_{\mathrm{p}}$ represent the cost of infection while the terms $\frac{A_{1}}{2} v_{1}^{2}, \frac{A_{2}}{2} v_{2}^{2}, \frac{A_{3}}{2} v_{3}^{2}$ represents the cost of the vaccination and drugs at the time $t$.

The goal is to find an optimal control $v_{1}{ }^{*}, v_{2}{ }^{*}$ and $v_{3}{ }^{*}$ such that,

$$
J\left(v_{1}{ }^{*}, v_{2}{ }^{*}, v_{3}{ }^{*}\right)=\min _{\Omega} J\left(v_{1}, v_{2}, v_{3}\right)
$$

where $\Omega=\left\{v_{1}(\mathrm{t}), v_{2}(t), v_{3}(t) \mid 0 \leq v_{1}(t), v_{2}(t), v_{3}(t) \leq 1\right.$ are measureable $\}$

Applying the Pontryagin's Maximum Principle, we have the following result

Theorem 3.5

There exists an optimal control $v_{1}{ }^{*}, v_{2}{ }^{*}, v_{3}{ }^{*}$ and the corresponding solution $\left(S_{n}^{*}, S_{p}^{*}, \mathrm{E}^{*}, I_{n}^{*}, I_{p}^{*}, \mathrm{R}^{*}\right)$ of the system (2) that minimizes $\mathrm{J}\left(v_{1}, v_{2}, v_{3}\right)$ over $\Omega$. Furthermore there exist ad joint functions $\lambda_{s_{n}}, \lambda_{s_{p}}, \lambda_{E}, \lambda_{I_{n}}, \lambda_{I_{p}}, \lambda_{R}$ such that,

$$
\begin{gathered}
\frac{d \lambda_{s_{n}}}{d t}=\left(c\left(1-v_{1}\right)+\beta_{n}\left(I_{p}+I_{n}\right)\left(1-v_{2}\right)-(a+d)\right) \lambda_{s_{n}}-\alpha \lambda_{s_{p}}-\lambda_{E} \beta_{n}\left(I_{p}+I_{n}\right)\left(1-v_{2}\right) \\
\frac{d \lambda_{s p}}{d t}=\left(\beta_{p}\left(I_{p}+I_{n}\right)\left(1-v_{2}\right)+d\right) \lambda_{s_{p}}-\lambda_{E}\left(\beta_{p}\left(I_{p}+I_{n}\right)\left(1-v_{2}\right)\right) \\
\frac{d \lambda_{E}}{d t}=-\mathrm{B}+(\omega+\mu+d) \lambda_{E}-\omega \lambda_{I_{p}} \\
\frac{d \lambda_{I p}}{d t}=-\mathrm{D}+\beta_{n} S_{n}\left(1-v_{2}\right) \lambda_{s_{n}}+\beta_{p} S_{p}\left(1-v_{2}\right) \lambda_{s_{p}}-\beta_{n} S_{n}\left(1-v_{2}\right) \lambda_{E}-\beta_{p} S_{p}\left(1-v_{2}\right) \lambda_{E}+(\Omega+\mu+d) \lambda_{I_{p}} \\
\frac{d \lambda_{I_{n}}}{d t}=-\mathrm{C}+\beta_{n}\left(1-v_{2}\right) \lambda_{S_{n}}+\beta_{p} S_{p}\left(1-v_{2}\right) \lambda_{s_{p}}-\beta_{n} S_{n}\left(1-v_{2}\right) \lambda_{E}-\beta_{p} S_{p}\left(1-v_{2}\right)+\left(v_{3}+\mathrm{d}\right) \lambda_{I_{n}}-v_{3} \lambda_{R} \\
\frac{d \lambda_{R}}{d t}=-\gamma \lambda_{s_{n}}+(\gamma+\mathrm{d}) \lambda_{R}
\end{gathered}
$$

With transversality conditions

$$
\lambda_{i}(T)=0, i=1,2,3,4,5,6
$$

The control triplet $v_{1}{ }^{*}, v_{2}{ }^{*}$ and $v_{3}{ }^{*}$ satisfies the optimality condition

$$
\begin{gathered}
v_{1}^{*}=\min \left(1, \max \left(0, \frac{c \lambda_{s n} S_{n}}{A_{1}}\right)\right) \\
v_{2}^{*} \min \left(\begin{array}{c}
\beta_{n} S_{n}\left(I_{p}+I_{n}\right)\left(\lambda_{E}-\lambda_{s n}\right)+\beta_{p} S_{p}\left(I_{p}+I_{n}\right) \\
1, \max \left(0, \frac{x\left(\lambda_{E}-\lambda_{s p}\right)}{A_{2}}\right) \\
v_{3}^{*}=\operatorname{Min}\left(1, \max \left(0, \frac{\left.\lambda_{R}-\lambda_{\text {In }}\right) I_{n}}{A_{3}}\right)\right.
\end{array}\right)
\end{gathered}
$$

Proof:

The proof follows with the Hamiltonian $\mathrm{H}$, given by

$$
\mathrm{H}=\mathrm{BE}+\mathrm{CI}_{\mathrm{n}}+\mathrm{DI}_{\mathrm{p}}+\frac{A_{1}}{2} v_{1}^{2}+\frac{A_{2}}{2} v_{2}^{2}+\frac{A_{3}}{2} v_{3}^{2}+\sum_{i=1}^{6} \lambda_{i} f_{i}
$$

Where $f_{i}$ for $i=1,2,3, \ldots \ldots, 5$ are the right hand sides of the model (2).

The optimal control $v_{1}^{*}, v_{2}^{*}$ and $v_{3}^{*}$ can be solved from optimality conditions

$$
\frac{\partial H}{\partial v_{1}}=0, \frac{\partial H}{\partial v_{2}}=0, \frac{\partial H}{\partial v_{3}}=0
$$

Where

$$
\begin{gathered}
\frac{\partial H}{\partial v_{1}}=c \lambda_{s n} S_{n}-\mathrm{A}_{1} v_{1}=0 \\
\frac{\partial H}{\partial v_{2}}=\beta_{n} S_{n}\left(I_{p}+I_{n}\right)\left(\lambda_{E}-\lambda_{s n}\right)+\beta_{p} S_{p}\left(I_{p}+I_{n}\right)\left(\lambda_{E}-\lambda_{s p}\right)- \\
\mathrm{A}_{2} v_{2}=0
\end{gathered}
$$




$$
\frac{\partial H}{\partial v_{3}}=\left(\lambda_{R}-\lambda_{I n}\right) I_{n}-\mathrm{A}_{3} v_{3}=0
$$

Hence the optimal effort necessary to control the disease is

$$
\begin{gathered}
v_{1}^{*}=\frac{c \lambda_{s n} S_{n}}{A_{1}} \\
v_{2}^{*}=\frac{\beta_{n} S_{n}\left(I_{p}+I_{n}\right)\left(\lambda_{E}-\lambda_{s n}\right)+\beta_{p} S_{p}\left(I_{p}+I_{n}\right)\left(\lambda_{E}-\lambda_{s p}\right)}{A_{2}} \\
v_{3}^{*}=\frac{\left(\lambda_{S n}-\lambda_{I n}\right) I_{n}}{A_{3}}
\end{gathered}
$$

\section{Discussion}

We explore the model with the following control measures; efficacy of vaccine therapy in preventing new infection $\left(\mathrm{v}_{1}\right)$, efficacy of vaccine in inhibiting the virus strain $\left(\mathrm{v}_{2}\right)$ and the effort to increase the number of recovered birds $\left(\mathrm{v}_{3}\right)$, to study the effects of control practices on transmission of Newcastle disease. This is done under the following scenarios to compare numerical results.

Strategy A: Optimal implementation of vaccine therapy ( $\mathrm{v}_{1}$ $\neq 0)$, efficacy of vaccines $\left(v_{2} \neq 0\right)$, and effort to increase the number of recovered birds $\left(\mathrm{v}_{3} \neq 0\right)$.

Strategy B: Optimal implementation of vaccine therapy $\left(\mathrm{v}_{1}\right.$ $\neq 0)$ and efficacy of vaccines $\left(v_{2} \neq 0\right)$ without any effort to increase the number of recovered birds $\left(\mathrm{v}_{3}=0\right)$

Strategy C: Optimal implementation of vaccine therapy $\left(\mathrm{v}_{1}\right.$ $\neq 0$ ) and optimal effort to increase the number of recovered birds $\left(v_{3} \neq 0\right)$ without efficacy of vaccines $\left(v_{2}=0\right)$

Strategy D: Optimal effort to increase the number of recovered birds $\left(v_{3} \neq 0\right)$ and efficacy of vaccines $\left(v_{2} \neq 0\right)$ without optimal implementation of vaccine therapy $\left(\mathrm{v}_{1}=0\right)$.

Strategy E: Optimal effort to increase the number of recovered birds $\left(\mathrm{v}_{3} \neq 0\right)$, withv $\mathrm{v}_{1}=\mathrm{v}_{2}=0$.

Strategy F: Optimal efficacy of vaccines $\left(\mathrm{v}_{2} \neq 0\right)$, with $\mathrm{v}_{1}=\mathrm{v}_{3}=0$.

Strategy G: optimal implementation of vaccine therapy $\left(v_{1} \neq\right.$ 0 ), with $\mathrm{v}_{2}=\mathrm{v}_{3}=0$

The latently infected, infected productive and infective nonproductive birds with and without control are plotted using the parameters values as in Table 2 below.

Numerical Solution of the ND

Table 2. Parameter values for Newcastle disease

\begin{tabular}{lll}
\hline Parameter & Estimated & References \\
\hline$\mu$ & 0.6 & Bornall et al (2015) \\
$\beta$ & 0.1 & Sharma et al (2015) \\
$\mathrm{d}$ & 0.5 & Qin \&Zheng (2016) \\
$\mathrm{p}$ & 100 & Elizabeth (2016) \\
$\tau$ & 0.033 & Elizabeth (2016) \\
$\nu$ & 0.026 & Elizabeth (2016) \\
$\delta$ & 0.026 & Elizabeth (2016) \\
\hline
\end{tabular}

\begin{tabular}{lll}
\hline Parameter & Estimated & References \\
\hline$\Omega$ & 0.033 & Hugo et al (2016) \\
$\Omega$ & 0.182 & Hugo et al (2016) \\
$\alpha$ & 0.01 & Hugo et al (2016) \\
$\gamma$ & 0.16 & Hugo et al (2016) \\
$\mathrm{d}$ & 0.2 & Hugo et al (2016) \\
$\beta_{n}$ & 0.03 & Hugo et al (2016) \\
$\beta_{p}$ & 0.003 & Hugo et al (2016) \\
\hline
\end{tabular}

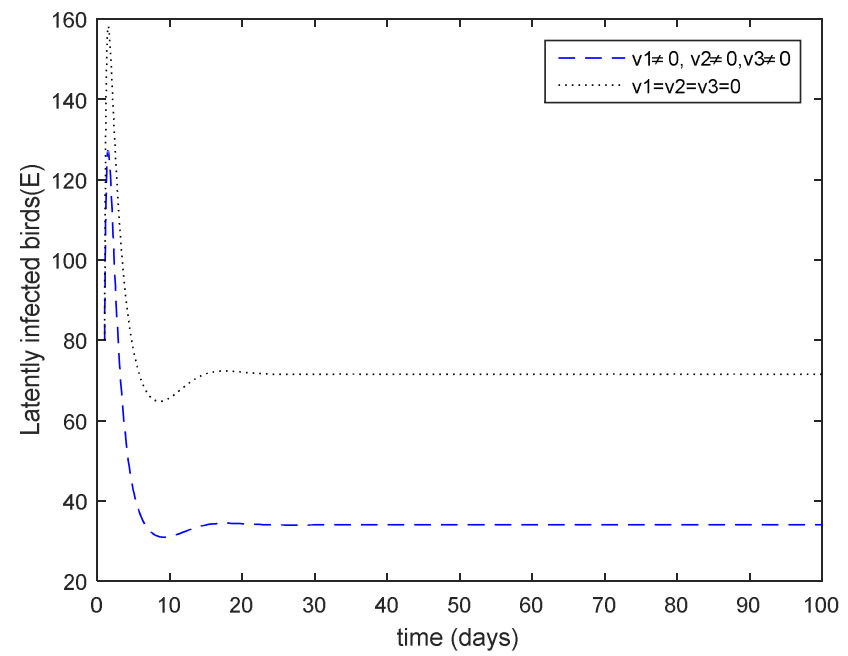

Figure 2. A plot representing the population of latently infected birds with and without control.

In Figure 2, we observe that in absence of any control measure, the number of latently infected birds will increase rapidly from the initial population size of 80 to 160 birds within 1-3 days, whereas in the presence of control measures the population size will reduces to about 30 birds and goes to a stable state. This shows that the control measures are effective.

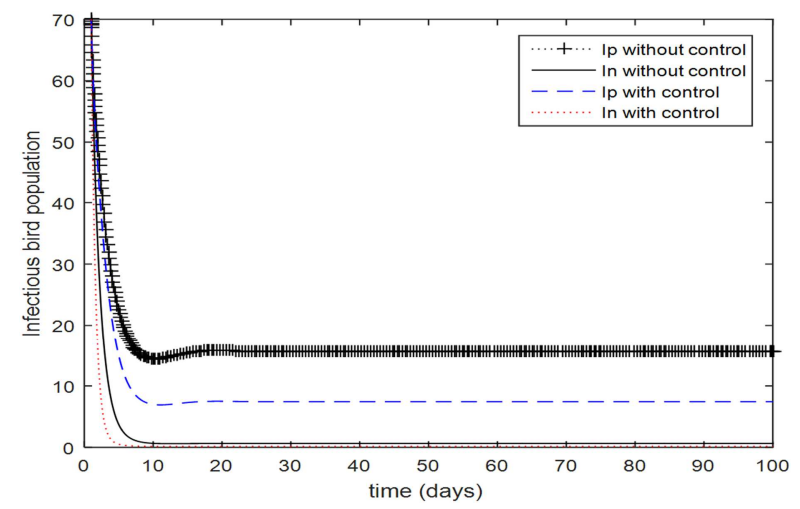

Figure 3. A plot representing the population of infectious birds with and without control.

The effect of the three control measures on the infectious classes can be seen in Figure 3. The number of non-productive infectious birds reduces to zero with control whereas the number of infectious productive reduces to about 8 birds and goes to its stable state when control is applied. This shows that the application of all three control measures tends to be more effective in the non- productive infectious bird population. 


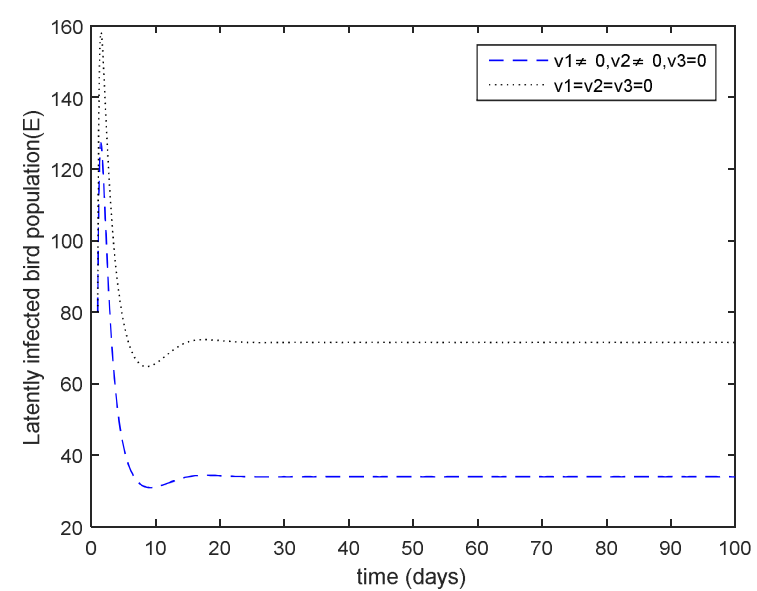

Figure 4. A plot representing the population of latently infected birds with optimal vaccination therapy $\left(v_{1}\right)$ and efficacy of vaccines $\left(v_{2}\right)$ without optimal effort to increase the number of recovered birds $\left(v_{3}\right)$.

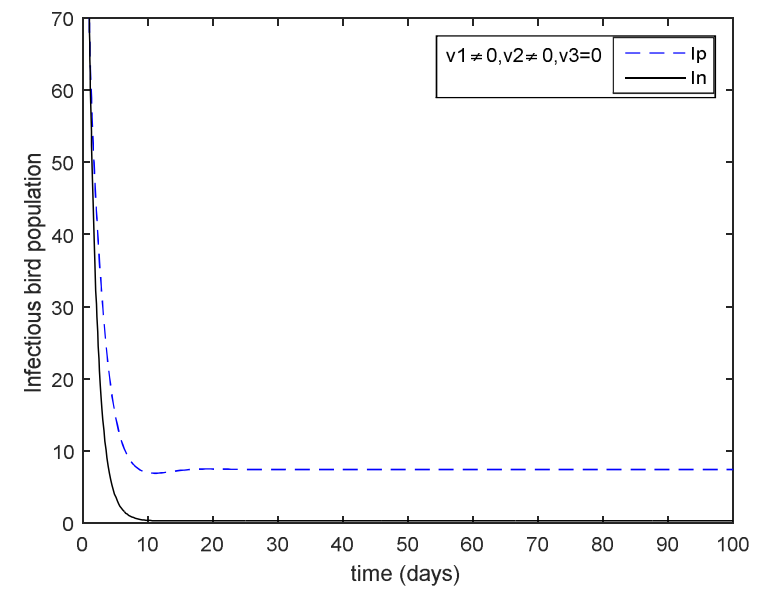

Figure 5. A plot representing the infectious bird population with optimal vaccination therapy $\left(v_{1}\right)$ and efficacy of vaccines $\left(v_{2}\right)$ without optimal effort to increase the number of recovered birds $\left(v_{3}\right)$.

From Figures 4 and Figure 5, the combination of efficient vaccination therapy and optimal efficacy of the vaccines are significantly more effective in the infectious productive birds population, since the combination reduces the population size of the birds to zero with 9-10 days.

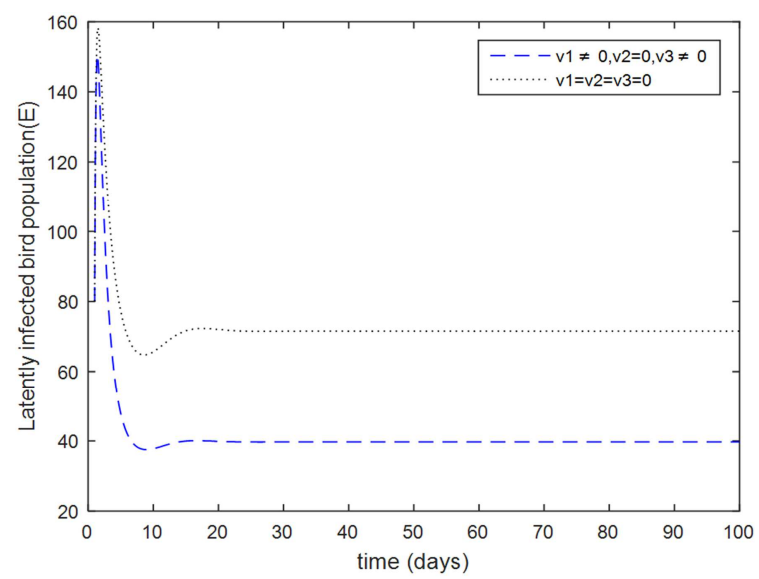

Figure 6. A plot representing the latently infected bird population with optimal vaccination therapy $\left(v_{1}\right)$ and optimal effort to increase the number of recovered birds $\left(v_{3}\right)$ without optimal efficacy of vaccines $\left(v_{2}\right)$.

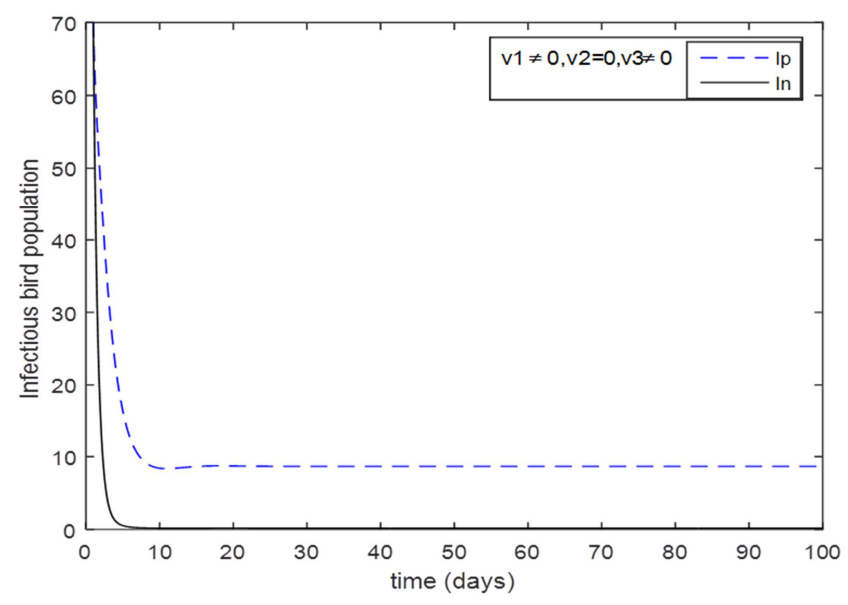

Figure 7. A plot representing the infectious bird population with optimal vaccination therapy $\left(v_{1}\right)$ and optimal effort to increase the number of recovered birds $\left(v_{3}\right)$ without optimal efficacy of vaccines $\left(v_{2}\right)$.

It is observed from Figure 6 that with this combination does not much effect on the latently infected birds at the early days of the outbreak, since the population size increases to about 130 before it starts reducing to its stable state.

From Figure 7, the infectious non -productive bird reduces to zero whereas the productive infectious bird population reduces to 9 birds on the $9^{\text {th }}$ day of the disease outbreak.

From Figure 8 we see that optimal efficacy of the vaccine and effort to increase the number of recovered birds increases the number of latently infected birds population to about 129 at the early days of the infection whereas from Figure 9, the infectious productive birds reduces to 15 while the non productive birds reduces to zero.

The results from Figure 10 show clearly, the effect of vaccination therapy on the latently infected birds. We observe that this programme will reduce the number of latently infected birds even if it not done more often. From Figure 11, we observe that this programme has effect on the infectious classes especially the non-productive infectious bird population, which reduces to zero after about 4 days.

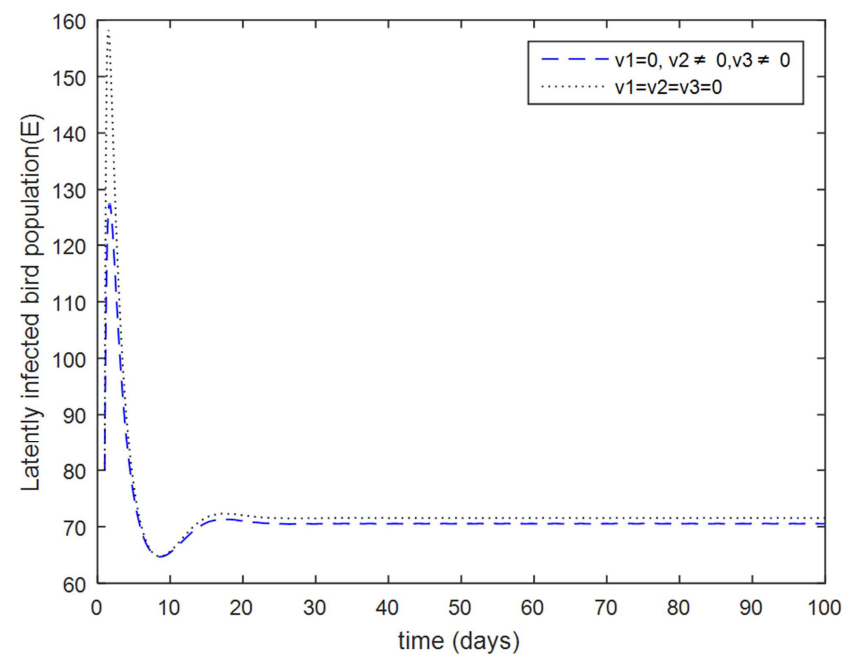

Figure 8. A plot representing the latently infected bird population with optimal efficacy of vaccines and optimal effort to increase the number of recovered birds $\left(v_{3}\right)$. without optimal vaccination therapy. 


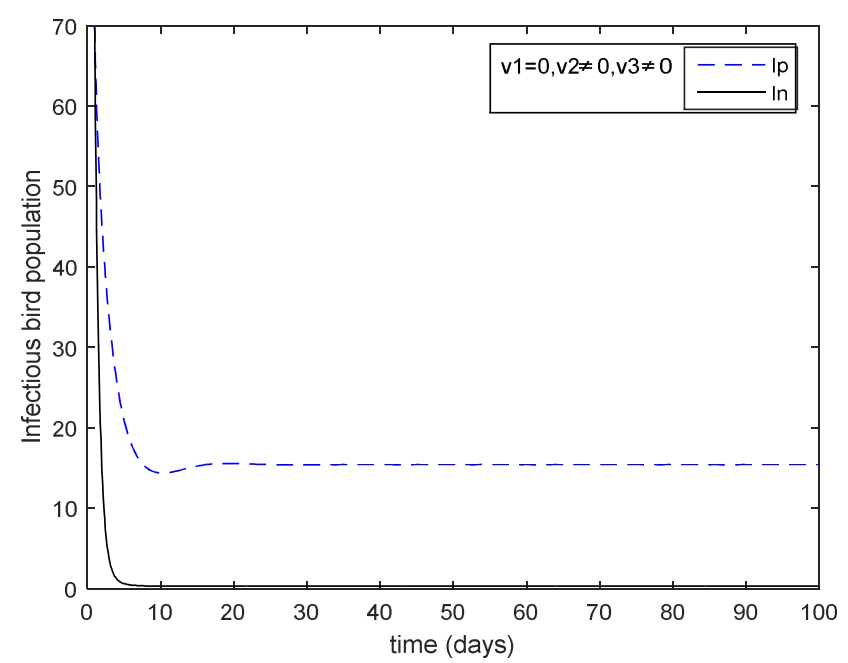

Figure 9. A plot representing the infected bird population with optimal efficacy of vaccines and optimal effort to increase the number of recovered birds $\left(v_{3}\right)$. without optimal vaccination therapy.

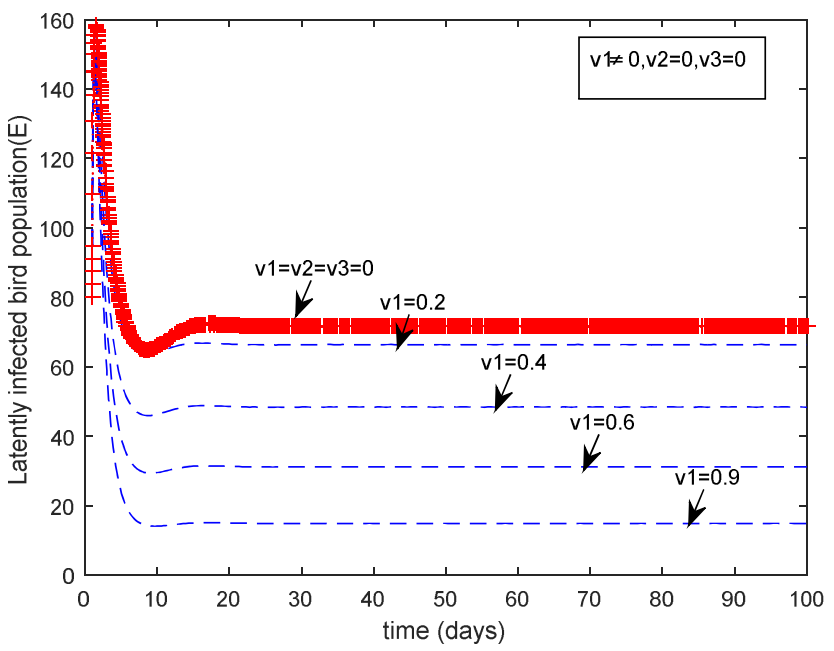

Figure 10. A plot showing the effect of only optimal vaccination therapy on latently infected bird population.

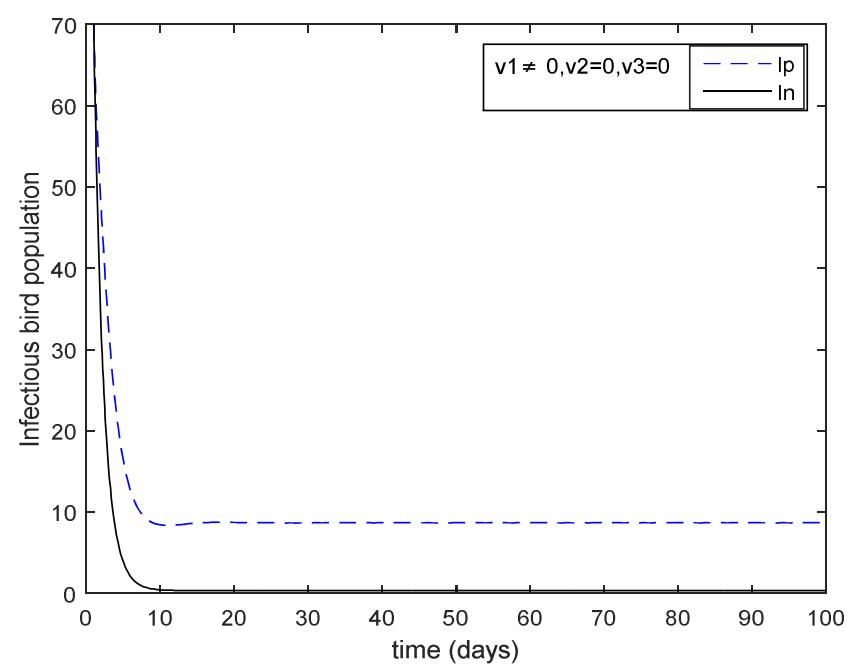

Figure 11. A plot showing the effect of only optimal vaccination therapy on the infectious bird population.

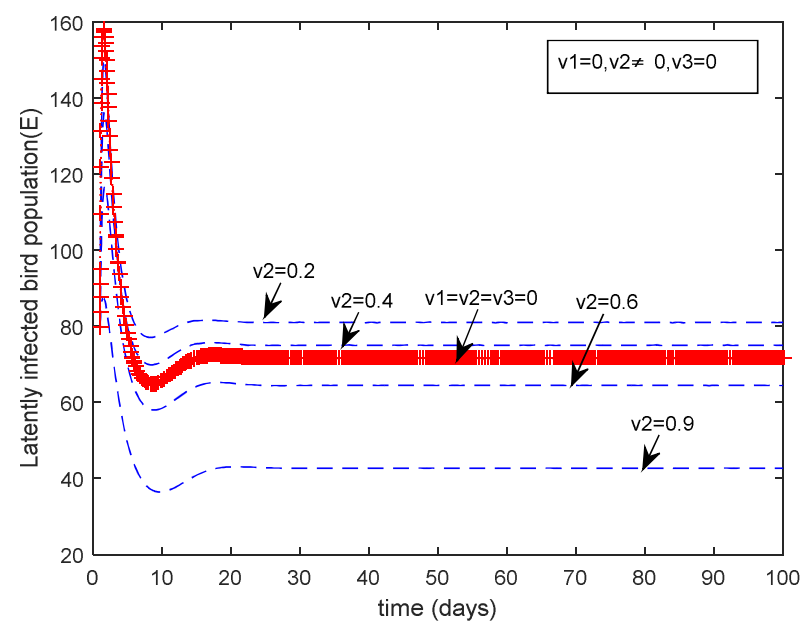

Figure 12. A plot showing the effect of vaccine efficacy on latently infected bird population.

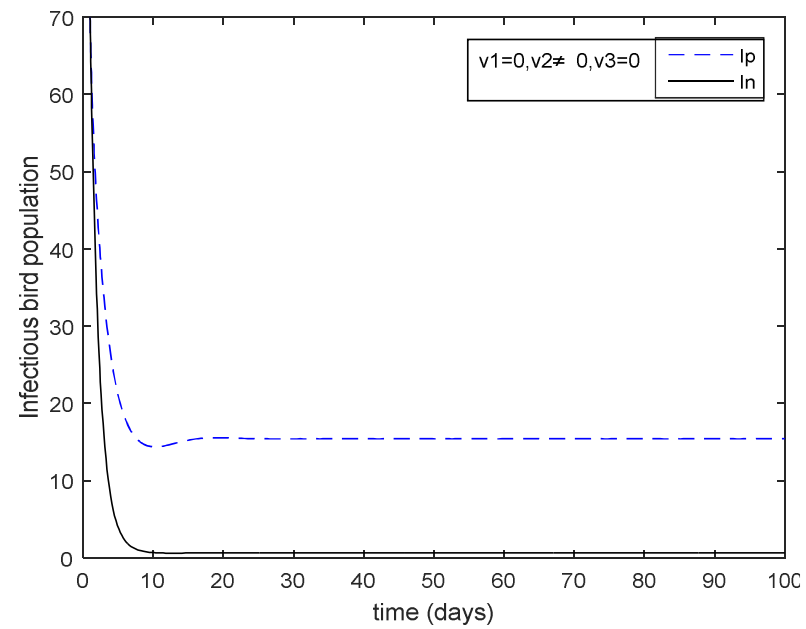

Figure 13. A plot showing the effect of vaccine efficacy on the infectious bird population.

The graph on Figure 12 shows that if the efficacy of the vaccine increases to about $60 \%$, the number of latently infected birds will fall below the number without control. From Figure 13 , the efficacy of the vaccine has more impact on the infectious non -productive bird than the infectious productive bird.

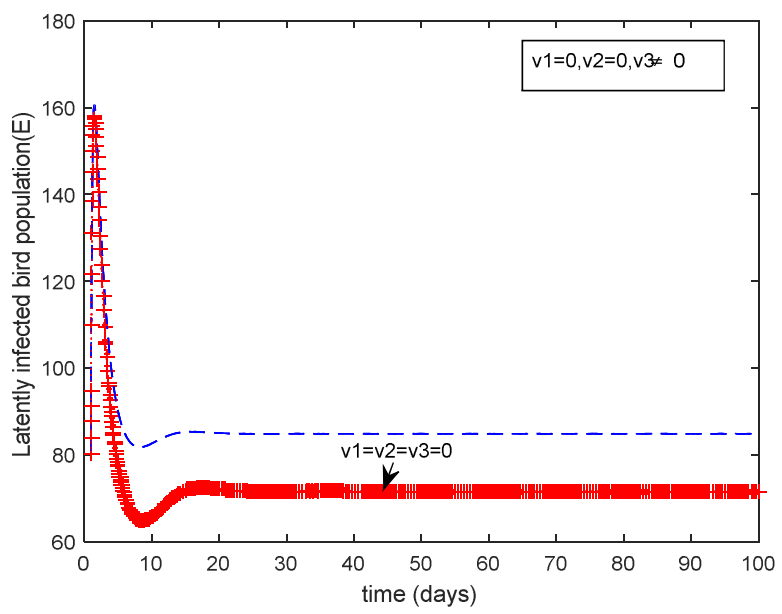

Figure 14. A graph showing the effect control $v_{3}$ on the latently bird population. 


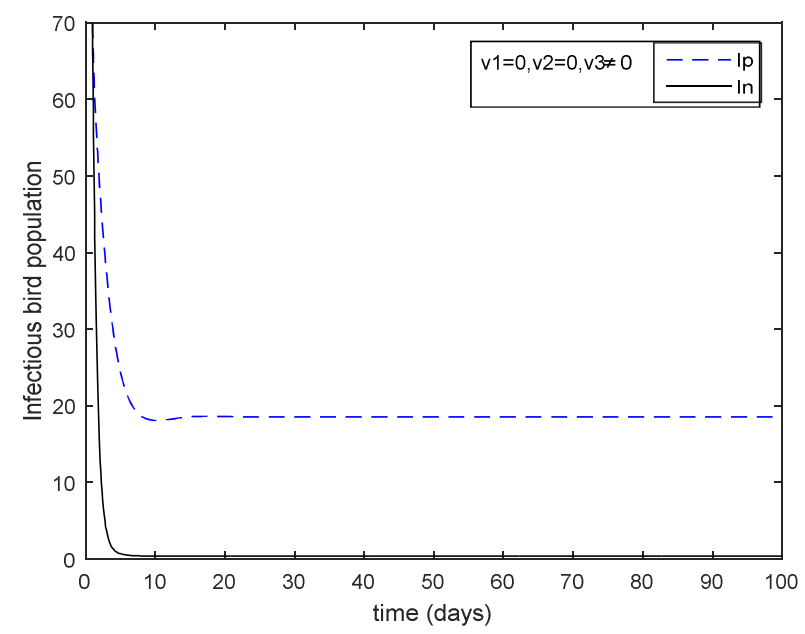

Figure 15. A graph showing the effect control $v_{3}$ on the infectious bird population.

It is evident from Figure 15 that as the number of infectious productive birds reduces as a result of disease induced death, it drops to below 20 and then stabilises. The number of nonproductive birds will drop to zero.

In this thesis, we considered the theoretical analysis of compartmental Newcastle disease (ND). The study is briefly summarised below;

Firstly, stability analysis was carried out using the Lyaponuv function theory and Lasslle's invariance principle for each of these disease models. Subsequently optimal control problems were formulated for the control models and was analysed using the pontryagin's maximum principle. Sensitivity analysis was also carried out to find out how important each model parameters are to the disease transmissions. This was done using the normalized forward- sensitivity index.

Finally, the results of the study were presented using numerical simulations for the disease models, for which each intervention strategies were discussed and results established.

\section{Conclusion}

The Newcastle disease model is divided into six compartments were we considered the productive and non productive bird populations. The DFE and EE is locally asymptotically stable if $\mathrm{R}_{0}<1$ and $\beta>\frac{c-\alpha-d}{I_{p}+I_{n}}$ respectively and is established to be globally asymptotically stable if $\mathrm{R}_{0}<1$ for $\mathrm{DFE}$ and $\mathrm{R}_{0}>1$ for $\mathrm{EE}$. The control strategies includes the control variable based on the efficiency of vaccine therapy $\left(\mathrm{v}_{1}\right)$, the efficacy of the vaccine $\left(v_{2}\right)$ and effort to increase the number of recovered birds. We observed from the numerical results, that the control strategies did not have much impact on the infectious non -productive birds but had high impact on the latently infected and infectious productive birds. The combination of effective revaccination program and optimal efficacy of the vaccines was found to reduce the number of infectious productive birds, hence will increase the level of bird productivity.

The work was motivated by the possibility that mathematical modelling could improve the understanding of the dynamics of this disease, particularly the impact of infection on poultry productivity. Based on the analysis of this study, we can conclude that poultry productivity can still be achieved even in the presence of perverse disease outbreak, if appropriate control measures are applied. Hence we recommend that control programs that follow the strategies stated for each of the diseases in this study, can be used effectively to prevent and reduce the spread of these diseases, in order to enjoy high poultry productivity in our poultry industries.

\section{References}

[1] Achoja, F. O., Ike, P. C., Akporhuarcho, P. O. (2010). Economics of Veterinary Services delivery among commercial poultry farmers in a market-driven economy: Evidence from Delta state, Nigeria. International Journal of Poultry Science 9, 1140-1145.

[2] Alabi, R. A., Tariuma, I. O., Onemolense, P. E. A., Mafimisebi, A., Isah, T. A., Esobhawa, A. O., Oviasogie, D. I. (2000). Risk management in poultry enterprises in Edo state insurance scheme proceedings of the $5^{\text {th }}$ Annual conference of Animal Science Association of Nigeria, Sept. 19-22, Portharcourt. pp 182-184.

[3] Alders, R. G., Bagnol, B., Costa, R. \& Young, M. P. (2012). Sustainable control of Newcastle Disease in village poultry. INFPD International Network for Family Poultry Development (FAO) GPFRP Note No. 05.

[4] Blake, D. P., Tomley F. M., (2014) Securing Poultry Production from the ever-present Eimeria Challenge; Trends in Parasitology, 30 (1): 12-19.

[5] Bowong, S., Alaoui, A. (2013) Optimal intervention strategies for tuberculosis; Communications in Nonlinear science and Numerical simulations, 18 (6), 1441-1453.

[6] Buonomo, B., Lotignola D., Vargas De-Leon, C. (2014) Qualitative analysis and optimal control of an epidemic model with vaccination and treatment; Mathematics and Computers in Simulation, 100 pp 88-102.

[7] Cassidy, L. R., Calistus, N. N. \& Mathew, H. B. (2015). Modelling the burden of poultry disease on the rural poor in Madagascar. Elsevier One Health, 1 (6065).

[8] Docherty, D. E., Friend M. (1999). Newcastle Disease. General Field procedures and diseases of Birds; Madison, Wis, USA: USGS-National Wildlife Health Center, pp 175-180.

[9] Dortmans, J. C., Peeters B. P., Koch G. (2012). Newcastle disease virus outbreaks: vaccine mismatch or inadequate application? Veterinary Microbiology 160 (1-2): 17-22. Doi: 10.1016/.j.vetmic.2012-05.003.

[10] Dortmans, J. C., Peeters, B. P., Koch, G. (2014). Field vaccinated chickens with low antibody titres, Showing equally insufficient protection against matching and non-matching genotypes of Virulent Newcastle disease virus; Veterinary Microbiology, $172 \quad(1-2), \quad 100-107 ; \quad$ doi: 10.1016/j.vetmic.2014.05.004.

[11] Newcastle disease transmission and illegal trade on a wild population of white-winged parakeetsin Peru: A modelling $\begin{array}{lllll}\text { approach. PLoS ONE } 11 & \text { (1): e0147517. doi: }\end{array}$ 10.1371/journal.pone.0147517. 
[12] Fangge, L., Peixian, L. (2011). ARMA model for predicting the number of new outbreaks of Newcastle disease during the month. Computer Science \& Automation Engineering (CSAE) 2011 IEEE International Conference. Doi: 10.1109/CSAE.2011.5952933.

[13] Fox, W. (2010). Tick-borne disease-risks and reality, Borreliosis and Associated disease Awareness UK 2010.

[14] Harrison, E. M. (2013). Epidemiology and evolution of vector borne disease. Department of Mathematical sciences, University of Bath.

[15] Hugo, A., Makinde, O. D., Karmar, S., Chibwana F. (2016). Optimal control and cost effectiveness analysis for Newcastle disease eco-epidemiological model in Tanzania. Journal of Biological dynamics Vol. 11, No 1, 190-209.

[16] Ibu, O. J., Adulugba A., Adeleke M. A., Tijjani A. Y. (2000). Activity of Newcastle disease and Infectious bursal disease viruses in ducks and guinea fowls in Jos area, Nigeria. Journal of Veterinary Sciences, vol. 2, pp 45-46.

[17] Jake, M. F., Jessia B. L., Vincent L. C., Andres J. G., Elizabeth A. H., Maia M., Craig, W. O. (2014). Optimal sampling strategies for detecting Zoonotic disease epidemics; (Article on maternal Health task force year 5); Available online at http://dx.doi.org/10.1371/journal.pibi.1003668.

[18] Jing, L., Xu D., Zang J., Xiao J., Wang H. (2010). The comparison of ARMA exponential Smoothing and seasonal Index model for predicting incidence of Newcastle Disease; World Automation Congress (WAC).

[19] Jos, C. F. M., Dortmas J. C., Koch G., Rottier P. J. M., Peters B. P. H. (2011). Virulence of Newcastle Disease virus: What is known so far? Veterinary Research 42: 122. doi: 10.1186/12979716, 42-122.

[20] Kapczynski, D. R., Afonso, C. L., Miller, P. J. (2013). Immune response to poultry to Newcastle disease virus. Dev. Comp. Immunology 41 (3): 447-533. doi: 10.1016/j.dci.2013.04.012.

[21] Karl, M. R. (2007). New methods for integrated models of animal disease control. Selected paper prepared for the 2007 American Agricultural Economics Association meetings, Portland.

[22] Linda, P. H. (2009). Epidemiology and characterization of Newcastle disease in small holder poultry in Mozambique; Available online at http:/epsilon.slu.se, ISSN 1652-8697.

[23] Ologbon, O. A. C., Ambatu O. I. (2012). Poultry enterprise combination among small-scale farmers in Ogun state, Nigeria: A technical sufficiency approach. Journal of Agricultural Veterinary Science, 4: 7-15.

[24] Oluwayelu, D. O., Adeiyi A. I., Olaniyian I., Ezewele P., Oluwasanmi A. (2014). Occurrence of Newcastle disease and infectious bursal disease virus antibodies in double-spurred Francolins of Nigeria; Journal of veterinary medicine 106898. Doi: $10.1155 / 2014 / 106898$.

[25] Omame, A., Okuonghae, D., Umana, R. A., Inyama, S. C., (2020). Analysis of a co-infection model for HPV-TB, Applied Mathematical Modelling, 77: 881-901

[26] Senne, D. A., King, D. J., Kapczynski D. R. (2004). Control of Newcastle disease by vaccination. Dev. Biology (Basel), 119: 165-170.

[27] Sharma, S., Samanta G. P. (2015). Stability analysis and optimal control of an epidemic model with vaccination. International Journal of Biomathematics, vol. 8, issue 03 .

[28] Udofia, Ekere Sunday and Inyama, Simeon Chioma (2011) Mathematical Modeling of the Transmission Dynamics of Fowl Pox in Poultry, Journal of Modern Mathematics and Statistics 5 (5-6), Pp 106-111.

[29] Udofia, Ekere Sunday and Inyama, Simeon Chioma (2012) Application of Optimal Control to the Epidemiology of Fowl Pox Transmission Dynamics in Poultry, Journal of Mathematics and Statistics 8 (2), Pp. 248-2012.

[30] Van, B. M., Bouwa A., Fabri T. H., Katsma E., Hartog L., Koch G. (2008). Herd immunity to Newcastle disease virus in poultry by vaccination. Journal of Avian Pathology 37 (1): 1-5. doi: 10.1080/03079450701772391. 